\title{
A review of the clinical evidence related to early treatment of elevated LDL for cardiovascular primary prevention
}

\section{Kori Sauser, ${ }^{1}$ Deborah A Levine, ${ }^{2,3}$ Rodney A Hayward ${ }^{2,3}$}

10.1136/ebmed-2015-110170

${ }^{1}$ Department of Emergency Medicine, Massachusetts General Hospital, Boston, Massachusetts, USA ${ }^{2}$ Department of Medicine, University of Michigan, Ann Arbor, Michigan, USA

${ }^{3}$ Center for Clinical Management Research, Ann Arbor Veterans Affairs Hospital, Ann Arbor, Michigan, USA

Correspondence to:

Kori Sauser,

Department of Emergency Medicine, Massachusetts General Hospital, Zero Emerson Place, Suite 3B, Boston, MA 02114, USA;

ksauser@mgh.harvard.edu

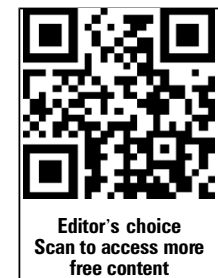

\section{(S) Linked}

- http://dx.doi.org/10.1136/ ebmed-2015-110257

\section{Abstract}

Background The American College of Cardiology/ American Heart Association updated cholesterol treatment guidelines dropped treatment recommendations based on elevated low-density lipoprotein (LDL) levels. Yet some experts cite the benefit of early statins in patients with elevated LDL for preventing atherosclerosis. We sought clinical evidence for this early LDL treatment hypothesis.

Methods and results A review of the clinical evidence examining the relationship between LDL reduction and outcomes (excluding LDL >190). We found three arguments proposed in the literature citing clinical evidence supporting the early LDL treatment hypothesis: (1), lower risk patients get relatively more primary prevention benefit from statins than higher risk patients, (2), statins demonstrate a legacy effect with prolonged risk reduction even after stopping treatment, and (3), genetic studies illustrate the benefit of lifelong LDL reduction for lowering CV risk. A review of the primary evidence found little clinical evidence supporting the first two arguments, but strong grade $\mathrm{B}+$ evidence for the third. However, we found no evidence for or against whether intervening before 10-year risk exceeds 7.5-12.5\% would result in substantial incremental net clinical benefit. If early intervention is practiced, evidence to date suggests that overall $\mathrm{CV}$ risk should be the primary indication.

Conclusions We found consistent grade B+ evidence that the effectiveness of LDL reduction on risk reduction will increase over time, however, we found no clinical evidence for or against whether starting before 10-year $\mathrm{CV}$ risk is $7.5-12.5 \%$ provides substantive additional net patient benefit, and grade A- evidence that elevated ageadjusted CV risk should be the primary indication for early treatment, but found no evidence for or against whether degree of LDL elevation should be a secondary factor. Additional clinical research is needed, especially with regard the long-term safety of statins and how long it takes for LDL reduction to reach full effectiveness.

\section{Introduction}

In November 2013, the American College of Cardiology and American Heart Association (ACC/AHA) published updated guidelines for the treatment of blood cholesterol. ${ }^{1}$ These guidelines recommend basing statin treatment for primary prevention on overall 10-year cardiovascular (CV) risk and no longer recommend treating based on specific low-density lipoprotein (LDL) levels unless it is very high $(\mathrm{LDL}>190 \mathrm{mg} / \mathrm{dL}) .^{2}{ }^{3}$ This decision was grounded in high-quality, randomised, controlled trial (RCT) evidence that one's pre-treatment LDL has no measurable impact on a statin's relative risk reduction (RRR) over a 5-10-year period. ${ }^{1-3}$
However, not all guidelines have shifted to this approach, and some experts opposed the removal of treatment recommendations for elevated LDL from the new ACC/AHA guidelines. They objected to the guideline committee not considering the implications of the pathophysiological and observational evidence including subgroup analyses of RCTs. While RCTs only consider 5-6-year benefits, these experts argue that evidence demonstrates the advantage of early treatment of elevated LDL for prevention of atherosclerosis and longterm reduction in CV morbidity and mortality that is not achieved if treatment is delayed until 10-year CV risk is increased above a certain threshold (eg, 7.5\%). We call this hypothesis the 'early LDL treatment hypothesis.' However, there is no systematic review of the clinical evidence for the early LDL treatment hypothesis.

To address this gap, we reviewed the RCT and longitudinal, observational (ie, cohort, case-control, and quasi-experimental design including Mendelian randomisation) literature to examine the degree to which the early LDL treatment hypothesis is supported by clinical evidence. We were interested in evidence regarding three separable aspects of the hypothesis: (1) evidence for the general early treatment hypothesis (ie, that there is a point at which atherosclerosis has advanced to where statins are substantially less effective than if they had been started earlier), and (2) evidence for the clinical benefit-based early treatment critique (ie, there are specific patients for whom waiting until CV risk is above $7.5 \%$ will result in a clinically meaningful reduction in quality or length of life, that could be prevented by lipid lowering being initiated at a specified earlier point in time), and (3) evidence for the LDL-based early treatment critique (ie, that moderate LDL elevation (LDL between $145 \mathrm{mg}-190 \mathrm{mg} / \mathrm{dL}$ ) should be a major determinant of patient selection for early treatment). In this paper, we report the results of our literature review for clinical evidence supporting the early LDL treatment hypothesis. We report our findings by describing the evidence related to the early LDL treatment hypothesis.

\section{Methods}

\section{Literature search and review criteria}

We first sought all RCTs examining the association between LDL reduction and patient outcomes. We began by reviewing the references from the 2013 ACC/AHA Guidelines on the Treatment of Blood Cholesterol ${ }^{1}$ and the 2012 Cholesterol Treatment Trialists' (CTT) metaanalysis evaluating the effect of LDL lowering for low risk patients. ${ }^{2}$ In addition to RCTs, we sought clinical evidence from longitudinal observational designs including quasi-experimental, cohort and case-controlled studies. As a strong quasi-experimental design that has been well-applied in the cholesterol literature, we specifically sought Mendelian randomisation studies, which we 
identified using the PubMed search: ((“"cholesterol”[MeSH Major Topic]) AND "genetic" AND ("low density lipoprotein" OR "ldl cholesterol"[Title/Abstract])). One author screened the 675 returned articles' titles and abstracts. For each relevant article identified, we screened the references and checked related citations in PubMed, Scopus and Web of Science. Our results and discussion cite all articles that one of the authors found relevant to the early LDL treatment hypothesis.

\section{Expert solicitation}

Since the literature on lipids and CV disease is so vast, we also contacted experts in the field (based on guideline authorship and peer-reviewed publications) to solicit help identifying additional arguments and evidence. We especially targeted advocates of treating to LDL targets. We conducted a brief survey of a convenience sample of 19 experts identified by one of the authors (RAH). We informed the experts of the evidence we found, and requested any additional evidence related to early LDL treatment (solicitation email in online supplementary eAppendix 1). We sent a follow-up to non-responders after 1-week. Ten experts responded, identifying two additional articles and no additional arguments based on clinical evidence. ${ }^{4}$

\section{Focused data analysis}

From the clinical trials, we extracted cohorts' baseline risk of vascular events, pretreatment and post-treatment LDL levels in the control and treatment groups, groups' event rates, and early trial termination. We derived the control and treatment groups' relative and absolute risk rates for adverse events. For between-study comparisons, we standardised rates to absolute LDL reduction of $40 \mathrm{mg} / \mathrm{dL}$ and to $40 \% \mathrm{LDL}$ reduction (roughly the expected response to $40 \mathrm{mg}$ simvastatin). We used criteria outlined by the Agency for Healthcare Research \& Quality to assess the quality of observational and quasi-experimental studies. ${ }^{6}$ We included all arguments with any 'clinical evidence' (true or quasi-experiments, cohort, or case-control studies); none were omitted due to the quality of the clinical evidence. Arguments based on pathophysiology or opinion alone were excluded.

Notably, there are small discrepancies in the outcomes used in the ACC/AHA guidelines and other analyses. This does not create major problems for our discussion here, as we are focusing on RRR rather than absolute risk reduction (ARR), but this is an important point to recognise in reviewing this literature and we addressed it to the extent possible.

\section{Results}

Our review of the medical literature identified three arguments that cited clinical evidence related to the early LDL treatment hypothesis proposed by advocates of the hypothesis.

Argument \#1: Clinical trial evidence suggests that patients with lower CV risk have a higher RRR from statin therapy than those at higher risk. That is, statins are relatively more effective in lower risk patients than in higher risk patients.

Evidence: We found some clinical evidence to support this argument, which is largely based on a subgroup analysis of the 2012 CTT meta-analysis, but this evidence has several problems. ${ }^{2}$ The CTT investigators reported that there was a statistically significant trend for patients with a lower predicted 10-year CV risk having a greater RRR per absolute LDL reduction than patients with a higher 10 -year CV risk $(\mathrm{p}=0.003$ for trend). The CTT group interpreted these results to support a similar RRR per amount of absolute $L D L$ reduction for patients with low and high $\mathrm{CV}$ risk, ${ }^{2}$ but others have referenced this in support of the early LDL treatment hypothesis. ${ }^{7}$

These results do not necessarily apply to primary prevention patients or those with a 10-year risk less than $20 \%$. We discuss methodological issues with this analysis below, but if these results hold up with further analysis (see online supplementary eAppendix 2), this finding would add support to the general hypothesis-in that those with highly advanced disease may get less benefit. However, this analysis does not provide clinical evidence addressing the specific clinical critique (that treatment must begin before CV risk is 7.5-12.5\%), in that the relative risks are very similar in this analysis in all those with a 10-year CV risk <21-22\%.

The meta-analysis findings may result from observational differences between statin trials rather than true variation in statins' effect across patient groups: Briefly, the CTT analysis provides observational evidence because there was substantial between-trial differences in the amount of CV RRR demonstrated per LDL reduction. $^{8-27}$ In online supplementary eAppendix 2, we explain this in more detail and propose a statistically robust analytic approach ${ }^{28} 29$ to examine for withinstudy heterogeneity in RRR by CV risk and provide stronger experimental evidence to clarify whether statins' benefit for primary prevention differs between lower-CV risk patient and higher-CV risk patient groups.

Early study termination may bias results: Seven of the RCTs (contributing 60\% of the low-risk and moderate-risk participants) were terminated early for efficacy at interim analysis.9 121518212226 Early termination systematically over-estimates a treatment's RRR. The CTT authors included a subanalysis excluding five of these trials, ${ }^{9} 12 \quad 152126$ and reported that results were "qualitatively similar... (data not shown)." It is unclear whether these results were statistically significant as the authors concluded that the results support a similar RRR for patients with low and high CV risk. The analytic method discussed in online supplementary eAppendix 2 minimises the impact of between-trial differences and allows inclusion of early termination studies without biasing the heterogeneity of treatment effect analysis.

The method for LDL standardisation could bias estimates of statins' treatment effect: As discussed below, the issue of whether short-term statin benefits are a function of absolute or relative LDL reduction remains unresolved. Since the RCTs included in the CTT meta-analysis used statins of different potency, some way of standardisation across studies was necessary. Based on results from observational studies, the CTT group standardised using absolute LDL reduction. However, individual RCTs have not found a consistent association between baseline LDL (a predictor of absolute LDL reduction) and a given statin's RRR. This 
suggests that a statin's short-term RRR may be a function of per cent LDL reduction. ${ }^{2}{ }^{30}$ LDL's long-term impact on atherogenesis could still be based a function of absolute LDL level, which could explain the discrepancy.

If statin's short-term RRR is a function of relative LDL reduction, then standardising RCTs by absolute LDL reduction could potentially lead to lower-risk participants (eg, JUPITER participants) systematically appearing to have greater benefit per amount of LDL reduction than higher-risk participants (eg, 4S participants). However, this apparent difference results from a difference in standardisation methods (see table 1). For example, when LDL reduction is standardised as per cent reduction, JUPITER and $4 \mathrm{~S}$ participants receive almost identical benefit (RRRs of 35\% vs 34\% per $40 \%$ LDL reduction, respectively). We make this comparison to illustrate how profound this effect may be while we acknowledge that a re-examination of the individuallevel meta-analysis is needed to know the correct standardisation method (see online supplementary appendix).

Argument \#2: Statin trials demonstrate a legacy effect-that is, the CV risk of treated patients continues to be reduced even after the trial ends.

Evidence: Again, we found little evidence for this claim. The only statin follow-up study that documented that LDLs equalised quickly after the trial ended, the HPS, found no evidence of a legacy effect. The HPS investigators found that once the two arms' LDLs converged, the groups' hazard rates also equalised. ${ }^{31}$ This suggests that, although the benefits achieved during the trial were not reversed post-trial, continued treatment is needed to maintain lower CV risk. This contrasts with a true legacy effect, as found in follow-up of the UK Prospective Diabetes Study: the intervention group maintained a greater RRR in the follow-up period after groups' A1c values equalised. ${ }^{32}$ As a secondary prevention study, it could be argued that HPS is not the optimal study for this question. However, it is a large, rigorous study and all other follow-up studies either reported no statistical testing for a legacy effect or lacked evidence for when and if LDL levels equalised.

Argument \#3: Genetic studies illustrate that lifelong LDL reduction is associated with a much greater CV risk reduction than seen in statin trials.

Evidence: This is a strong argument for the general hypothesis with grade $\mathrm{B}+$ evidence supporting it (that there is a point in the pathophysiological disease process at which LDL lowering will be less effective in the long-run). These studies used Mendelian randomisation, a quasi-experimental design employing instrumental variable analysis. ${ }^{33}$ The studies compare individuals with very low LDL related to randomly inherited polymorphisms to individuals without these mutations to then estimate the causal effects of lifetime LDL reduction on long-term CV risk. The CV benefit from lower LDL is then extrapolated to the benefit of having statinlowered LDL. Meta-analyses of such studies have consistently reported a CV risk reduction per $\mathrm{mg} / \mathrm{dL}$ LDL reduction that is at least two times greater than statin trials and cohort studies such as Framingham. ${ }^{34-38}$ This evidence is frequently cited as demonstrating that lifelong LDL lowering results in much greater risk reduction, favouring earlier LDL reduction strategies. ${ }^{39}$

The genetic observational evidence appears quite strong, but does not address the specific clinical critiques (ie, that there is clinically significant benefit in starting a statin before a patient's 10-year CV risk is 7.5$12.5 \%$, and LDL elevation should be the main factor in targeting patients for early treatment): We must consider the evidence related to: (1), is the estimated magnitude of $\mathrm{CV}$ benefit from long-term LDL reduction more accurately captured by the experimental RCT evidence or the genetic studies? And (2), if the degree of benefit is most accurately captured by the genetic studies, how does this support the early treatment hypothesis?

Like all observational study designs, the risk of confounding bias cannot be completely ruled out. The key assumption of genetic randomisation is that the polymorphism has no impact on the outcome (CV events) other than that mediated by the risk factor it affects (LDL). This cannot be proven, but the genetic studies in these meta-analyses are particularly strong in aggregate because the effect sizes per amount of LDL reduction have been consistent over 22 different polymorphisms. Furthermore, many of the strongly positive studies have been very large, making publication bias less likely. The design of these studies and consistency of results are quite robust for observational evidence.

If the genetic evidence represents robust observational evidence, then the alternative explanation is that the RCTs and other observational evidence systematically underestimate the magnitude of the relationship between lower LDL and CV risk reduction. In fact, the observational studies are prone to LDL measurement error where the genetic studies are not, which may lead to systematic underestimation of the true benefit of LDL reduction. Since there is no equivalent to an A1c for lipids, cohort analyses rely on patients' LDL measurements, which have substantial measurement error due to

Table 1 Standardisation of studies by absolute LDL reduction systematically misestimates CV RRR*

\begin{tabular}{lllllll}
\hline & $\begin{array}{l}\text { Baseline LDL } \\
(\mathrm{mg} / \mathrm{dL})\end{array}$ & $\begin{array}{l}\text { Treated LDL } \\
(\mathrm{mg} / \mathrm{dL})\end{array}$ & $\begin{array}{l}\text { Absolute LDL } \\
\text { reduction }(\mathrm{mg} / \mathrm{dL})\end{array}$ & $\begin{array}{l}\text { Proportional LDL } \\
\text { reduction }(\%)\end{array}$ & $\begin{array}{l}\text { CV RRR per 40 mg/dL } \\
\text { LDL reduction }\end{array}$ & $\begin{array}{l}\text { CV RRR per 40\% } \\
\text { LDL reduction }\end{array}$ \\
\hline JUPITER & 108 & 55 & 53 & 49 & 0.33 & 0.35 \\
SSSS & 188 & 122 & 66 & 35 & 0.18 & 0.34 \\
\hline
\end{tabular}

*This illustrates the difference between standardising trials based on absolute versus per cent reduction. JUPITER, a study with lower-risk low-LDL participants appears to have much greater benefit per amount of absolute LDL reduction (RRR 33\%) than 4S, a study with higher-risk higher-LDL participants (RRR 18\%). One might conclude that lower risk participants receive more benefit per LDL reduction than high-risk participants. However, when standardised as per cent reduction, JUPITER and $4 \mathrm{~S}$ participants receive almost identical relative CV benefit (RRR of $35 \%$ vs $34 \%$, respectively).

$\mathrm{CV}$, cardiovascular; LDL, low-density lipoprotein; RRR, relative risk reduction. 
laboratory error, day-to-day and year-to-year biological variation. This leads to regression dilution bias, which systematically underestimates the 'true' association between the measured risk factor and the outcome. Therefore, we expect traditional cohort analyses to underestimate the true association between LDL level and $\mathrm{CV}$ risk. ${ }^{40}{ }^{41} \mathrm{~A}$ few analyses have statistically accounted for this, ${ }^{4}{ }^{5}$ but most have not. In the online supplementary eAppendix, we outline methods to adjust for this systematic underestimation. ${ }^{40}{ }^{41}$ The genetic studies, in contrast, are protected from such measurement error by using individuals' inherited polymorphisms in aggregate and comparing the average LDLs in those with and without the polymorphism. The group average LDL measure used in genetic studies should more accurately reflect each group's cumulative LDL burden than periodic LDL measures.

Yet if the genetic studies represent a more accurate estimate of LDL's true impact on CV risk, published evidence still does not allow us to determine how long LDL must be reduced to achieve full efficacy. More specifically, the genetic studies do not help us with the two specific critiques: (A) the specific clinical critique, that waiting until CV risk is above 7.5\% is too late for some patients to receive the full clinically meaningful benefit of lipid lowering, and (B) the LDL-based early treatment critique, that if earlier treatment does produce clinically significant net benefit, then moderate LDL elevation (145-190 mg/dL) should be a primary determinant for early treatment.

First, we lack evidence for determining whether there are specific patients for whom waiting until CV risk is greater than $7.5 \%$ to initiate lipid lowering treatment will result in a clinically meaningful reduction in quality or length of life (the specific clinical critique). For example, if many years of LDL reduction are necessary in order to reach full efficacy (the maximum treatment RRR), then the opportunity for much of the potential benefit of LDL reduction could be lost if LDL lowering is delayed past some yet-to-be-determined point in CV pathogenesis. Then it is important to determine how the genetic evidence applies to the two specific questions: whether CV RRR increases over the 4-5-year period of statin trials and whether long-term effects of LDL reduction on CV events are a function of relative or absolute LDL reduction. We could not find an accurate analysis of whether CV RRR increases over the 4-5-year period of statin trials (see online supplementary eAppendices 2 and 4 for further discussion of how these two points may be addressed with the CTT data).

The recent Framingham analysis suggests that the cumulative effect of LDL is in fact an important independent risk factor. ${ }^{42}$ This new evidence is very important as it suggests that we can more accurately estimate $\mathrm{CV}$ risk by using cumulative LDL rather than a single given LDL level at a given point in time, and it also provides additional evidence for the general early treatment hypothesis. However, this important evidence does not directly address the two clinical critiques because it provides no evidence that cumulative LDL directly modifies RRR or that long-term CV morbidity and mortality will be substantively improved by initiating treatment before CV risk is around 10\%. It does suggest that tracking cumulative LDL burden could aid predicting CV risk. Another report found that initiation of statins early in life for those with familial hyperlipidaemia led to decreased carotid intima-media thickness at follow-up, yet did not include any clinical end points. ${ }^{43}$ We could find no evidence to guide how early statin intervention should occur or how safe long-term statin therapy would need to be to justify earlier intervention, assuming that the general early treatment hypothesis is correct.

\section{Discussion}

In our literature review for clinical evidence supporting the early LDL treatment hypothesis, we found three arguments that cited clinical evidence to support of the hypothesis: (1), lower risk patients get relatively more primary prevention benefit from statins than higher risk patients, (2), there is a legacy effect illustrated in the statin trials with continued benefit after trial conclusion, and (3) the genetic studies illustrate a greater magnitude of CV benefit with lifelong LDL reduction (box 1). We found the clinical evidence in support of the first two arguments to be weak, however, in the online supplementary appendix we propose analyses of existing data that could more rigorously evaluate these questions. The genetic studies provide the strongest support for the general early treatment hypothesis, but we could find almost no direct evaluation of the two important clinical critiques: whether intervention before CV risk is around $10 \%$ provide clinically substantive incremental benefit and whether degree of LDL elevation within the commonly observed range should play a major role in early treatment decision-making. Our conclusions are summarised below.

Conclusion \#1: There is strong grade B+ evidence for the general early treatment hypothesis.

The genetic studies and the recent evidence on the importance of cumulative LDL provide strong clinical evidence of the general hypothesis.

Conclusion \#2: There is no clinical evidence for or against the clinical benefit-based early treatment critique (that there is clinically significant net benefit from treating selected patients before their 10-year CV risk is above $7.5-12.5 \%$ ).

We found virtually no evidence assessing the potential absolute lifetime incremental benefits of initiating a statin earlier than currently recommended in the ACC/ AHA guidelines. ${ }^{44}$ Even though it is unclear how long it might take for statin therapy to reach its maximum RRR, modelling analyses could still be a useful approach of clarifying the circumstances under which earlier statin treatment could improve quality of life or life expectancy sufficiently to be worth increased treatment burdens and risks. These analyses could be similar to those examining the long-term benefits of glucose control or diabetes prevention. ${ }^{45}{ }^{46}$ In fact, grade B clinical evidence supporting a large ARR is preferable to grade A evidence for a very small ARR. Without some estimation of ARR, it is impossible to weigh potential benefits against the burden and long-term safety of an intervention, particularly when intervening on asymptomatic patients for events most likely to occur greater than 20 years in the future. And as early treatment leads to many more patients taking statins for 15-25 years 


\section{Box 1 Summary}

Objective: to identify clinical evidence in support of the early use of statins in patients with elevated low-density lipoprotein (LDL) levels ("the early LDL treatment hypothesis"). Methods: review the evidence examining the relationship between LDL reduction and outcomes. We identified any "clinical evidence' (true or quasi-experiments, cohort studies, or case-control studies), and excluded only those arguments based on pathophysiology or opinion alone.

Conclusions:

1 We found strong grade $B+$ clinical evidence supporting the general early treatment hypothesis-that there is a point at which atherosclerosis has progressed to a point that LDL reduction will have less long-term $\mathrm{CV}$ risk reduction than if done earlier.

2 We found no clinical evidence for or against the clinical benefit-based early treatment critique-that there is clinically significant net benefit from treating selected patients before their 10-year cardiovascular risk is above $7.5-12.5 \%$.

3 We found grade A- clinical evidence supporting that if earlier treatment is indicated, that elevated age-adjusted CV risk should be the primary indication. We found no evidence for or against the use of LDL level in isolation as an important secondary factor in targeting higher benefit patients.

4 We identified several specific research priorities that could help elucidate the above clinical issues, including the safety of long-term statin therapy, how long it takes for lipid lowering to reach full effectiveness, and whether the long-term effects of lipid lowering are a function of relative or absolute LDL reduction (see online supplementary eAppendix for details).

before the time when substantial ARR will occur, more rigorous postmarketing surveillance for up to 30 years for potential harms will be important. If 25-30 years of statin therapy results in even a minor change in the aging of muscles or the brain, it would easily overwhelm any potential long-term CV benefits. As demonstrated for glucose control, ${ }^{46}$ even a very small amount of treatment harm can outweigh benefits when those benefits are in the distant future.

Conclusion \#3: There is grade A- clinical evidence supporting that if earlier treatment is indicated, that elevated age-adjusted CV risk should be the primary indi- cation. We found no evidence for or against LDL level in isolation as a secondary factor in targeting higherbenefit patients.

There is currently no clinical evidence for or against whether moderate LDL elevation should be a major secondary factor in patient selection. Even if one's LDL level was a better predictor, it remains an isolated risk factor. Consider a 40-year-old women with a high $L D L$ but no other risk factors. Based on the Reynolds calculator, her 10-year CV risk is less than $1 \%$ at age 40 and if her risk factors other than age are unchanged, her CV risk at 60 will be only $2 \%$. In contrast, a man with low $L D L$ but several CV risk factors (eg, smoking and hypertension), is at high risk for rapid, premature atherogenesis. While his current $\mathrm{CV}$ risk (7\%) may be below the recommended treatment threshold, by age 60 his CV risk will increase to over 20\% even if BP and LDL levels do not increase. Clearly, a treatment decision based chiefly on LDL level instead of CV risk for these patients would be inconsistent with the clinical evidence currently available, as pointed out in several studies. ${ }^{47} 48$

It is beyond the scope of this review to consider which CV risk metric is appropriate if a clinician decides to adopt treatment earlier than proposed in the ACC/ AHA guidelines. Although the concept of "lifetime risk" has been proposed, such a measure can be heavily influenced by events very late in life, up to 35-50 years in the future. The key is to target those at particular risk for marked atherosclerosis formation over the next 10-15 years. This review is limited to the completeness of our literature review. Given the vastness of the epidemiological lipid literature, it is possible that we overlooked important evidence despite our extensive literature review. To minimise this risk, we contacted 19 experts in the field, oversampling proponents of early treatment of LDL. We informed them of our original review and analysis, and solicited additional evidence and arguments (see online supplementary appendix). Additionally, this paper assumes that LDL reduction is the main mechanism through which statins reduce $\mathrm{CV}$ risk, which is not universally accepted.

There are numerous papers discussing the physiological argument, that the known pathophysiology provides prima fascia evidence that atherosclerosis develops many years before CV risk increases. We did not address this common argument, however, as we limited our review to clinical evidence based on common evidencebased medicine principles. Of course, there are some who disagree with the principle that in order to make clinical recommendations, clinical evidence assessing the amount of net treatment benefits of a specific clinical recommendation is needed. Nevertheless, we do find grade $\mathrm{B}+$ clinical evidence to support the general early treatment hypothesis, and this supports the pathophysiological evidence without relying on it.

\section{Conclusion}

We found grade $\mathrm{B}+$ evidence supporting the general early treatment hypothesis-that there is some point at which atherosclerosis has progressed to the point that initiation of statin therapy will be significantly less effective over the long-term than if it had been started 
earlier in life. However, we found no evidence informing the specific clinical critique, or how early this intervention would need to start, or quantifying the potential benefits, risks, and treatment burden of initiating statins earlier than that proposed by the new AHA/ACC guidelines. Current evidence suggests that overall $\mathrm{CV}$ risk should still be the primary metric for targeting patients if early statin treatment, but we found no evidence for or against whether degree of LDL elevation should be an important secondary factor in those with LDL $<190 \mathrm{mg} / \mathrm{dL}$. Finally, we conclude that three questions should be a high priority for future clinical research: (1) If the relative benefit of statins increases over time, how much more would starting a statin before an individual's 10-year CV risk is 7.5-12.5\% reduce absolute risk of $\mathrm{CV}$ morbidity and mortality when compared with the current AHA/ACC guidelines?, (2) Is the short-term association between CV risk and cumulative LDL reduction a function of relative or absolute change in LDL?, and (3) Is there evidence of substantive harms from being on a statin for 25-35 years?

\section{Glossary}

General early treatment hypothesis: This hypothesis posits that there is some point at which atherosclerosis has advanced to a level that statins and other lipid lowering therapies are substantially less effective than if they had been started earlier.

Specific clinical critique: This hypothesis posits that there are specific patients for whom delay in lipid lowering therapy until CV risk is above $7.5 \%$ will result in a clinically meaningful reduction in quality or length of life, which could have been prevented by earlier initiation of treatment.

LDL-based early treatment critique: This hypothesis posits that for patients with moderate LDL elevation (145-190 mg/dL), LDL level in isolation should be a major determinant for initiation of early treatment.

Clinical evidence: True experiments, quasi-experiments, cohort or case-control studies in which meaningful clinical end points are examined.

Relative Risk Reduction (RRR): This is the proportion by which an exposure, such as a treatment, reduces a risk. In a clinical trial, the RRR is the difference in the event rates in the control and intervention arms divided by the rate in the control arm.

Mendelian randomisation: A type of quasiexperiment in which genetic polymorphisms are used to isolate effect of specific biological changes. For example, studies of gene mutations that result in low LDL levels have been used to try to estimate the impact of LDL reduction independent of the means by which it is reduced. The key assumption is that the polymorphism has no impact on the outcome (CV events) other than that mediated by the risk factor it affects, such as LDL in the above example.

Grade A evidence: Based on high-quality, welldesigned studies in representative populations, there is high certainty that the net benefit is substantial. ${ }^{49}$

Grade B evidence: Based on available evidence that may be limited by study quality, inconsistent findings, or generalisability, the available evidence indicates that there is net benefit, however, as more information becomes available the magnitude or direction of the effect could change and potentially alter the conclusion. $^{49}$

\section{Twitter Follow Kori Sauser at @k_sauser}

Acknowledgements The authors thank Elyse N Reamer, BS, University of Michigan, assisted with reference collection.

Contributors RAH conceived the study. KS and RAH conducted the review; and KS, DAL, and RAH all contributed to interpreting the data, reporting the findings, and drafting the manuscript.

Funding Financial support from The Department of Veterans Affairs Quality Enhancement Research Initiative (QUERI DIB 98-001), the Robert Wood Johnson Foundation, and the Michigan Center for Diabetes Translational Research supported by NIDDK of The National Institutes of Health (P60 DK-20572). DAL is funded by National Institutes of Health (NIH) grant K23 AG040278 and received support from NIH grant P30DK092926.

Competing interests None declared.

Provenance and peer review Not commissioned; externally peer reviewed.

- Additional material is published online only. To view please visit the journal online (http://dx.doi.org/10. 1136/ebmed-2015-110170).

\section{References}

1. Stone NJ, Robinson JG, Lichtenstein AH, et al. 2013 ACC/AHA guideline on the treatment of blood cholesterol to reduce atherosclerotic cardiovascular risk in adults: a report of the American College of Cardiology/American Heart Association Task Force on Practice Guidelines. Circulation 2014; 129(25 Suppl 2):S1-45

2. Mihaylova B, Emberson J, Blackwell L, et al, Cholesterol Treatment Trialists' (CTT) Collaborators. The effects of lowering LDL cholesterol with statin therapy in people at low risk of vascular disease: meta-analysis of individual data from 27 randomised trials. Lancet 2012;380:581-90.

3. Hayward RA, Krumholz HM. Three reasons to abandon low-density lipoprotein targets: an open letter to the Adult Treatment Panel IV of the National Institutes of Health. Circ Cardiovasc Qual Outcomes 2012;5:2-5.

4. Lewington S, Whitlock G, Clarke R, et al., Prospective Studies Collaboration. Blood cholesterol and vascular mortality by age, sex, and blood pressure: a meta-analysis of individual data from 61 prospective studies with 55,000 vascular deaths. Lancet 2007;370:1829-39.

5. Di Angelantonio E, Sarwar N, Perry P, et al., Emerging Risk Factors Collaboration. Major lipids, apolipoproteins, and risk of vascular disease. JAMA 2009;302:1993-2000.

6. Criteria to assess quality of observational studies evaluating the incidence, prevalence, and risk factors of chronic diseases (Text Version). Rockville, MD: Agency for Healthcare Research and Quality, 2009. http://www.ahrq.gov/news/events/conference/ 2009/shamliyan/index.html (accessed 8 Apr 2014).

7. Ridker $\mathrm{P}$, Wilson PF. A trial-based approach to statin guidelines. JAMA 2013;310:1123-4.

8. Wanner C, Krane V, März W, et al. Atorvastatin in patients with type 2 diabetes mellitus undergoing hemodialysis. $N$ Engl J Med 2005;353:238-48.

9. Downs JR, Clearfield M, Weis S, et al. Primary prevention of acute coronary events with lovastatin in men and women with 
average cholesterol levels: results of AFCAPS/TexCAPS. Air Force/Texas Coronary Atherosclerosis Prevention Study. JAMA 1998;279:1615-22.

10. Holdaas H, Fellström B, Jardine AG, et al. Effect of fluvastatin on cardiac outcomes in renal transplant recipients: a multicentre, randomised, placebo-controlled trial. Lancet 2003;361:2024-31.

11. ALLHAT Officers and Coordinators for the ALLHAT Collaborative Research Group. The Antihypertensive and Lipid-Lowering Treatment to Prevent Heart Attack Trial. Major outcomes in moderately hypercholesterolemic, hypertensive patients randomized to pravastatin vs usual care: the Antihypertensive and Lipid-Lowering Treatment to Prevent Heart Attack Trial (ALLHAT-LLT). JAMA 2002;288: 2998-3007.

12. Sever PS, Dahlöf B, Poulter NR, et al. Prevention of coronary and stroke events with atorvastatin in hypertensive patients who have average or lower-than-average cholesterol concentrations, in the Anglo-Scandinavian Cardiac Outcomes Trial-Lipid Lowering Arm (ASCOT-LLA): a multicentre randomised controlled trial. Lancet 2003;361:1149-58.

13. Knopp RH, d' Emden M, Smilde JG, et al. Efficacy and safety of atorvastatin in the prevention of cardiovascular end points in subjects with type 2 diabetes: the Atorvastatin Study for Prevention of Coronary Heart Disease Endpoints in non-insulin-dependent diabetes mellitus (ASPEN). Diabetes Care 2006;29:1478-85.

14. Fellström BC, Jardine AG, Schmieder RE, et al. Rosuvastatin and cardiovascular events in patients undergoing hemodialysis. N Engl J Med 2009;360:1395-407.

15. Colhoun HM, Betteridge DJ, Durrington PN, et al. Primary prevention of cardiovascular disease with atorvastatin in type 2 diabetes in the Collaborative Atorvastatin Diabetes Study (CARDS): multicentre randomised placebo-controlled trial. Lancet 2004;364:685-96.

16. Sacks FM, Pfeffer MA, Moye LA, et al. The effect of pravastatin on coronary events after myocardial infarction in patients with average cholesterol levels. Cholesterol and Recurrent Events Trial investigators. N Engl J Med 1996;335:1001-9.

17. Kjekshus J, Apetrei E, Barrios V, et al. Rosuvastatin in older patients with systolic heart failure. N Engl J Med 2007;357:2248-61.

18. [No authors listed]. Results of the low-dose $(20 \mathrm{mg})$ pravastatin GISSI Prevenzione trial in 4271 patients with recent myocardial infarction: do stopped trials contribute to overall knowledge? GISSI Prevenzione Investigators (Gruppo Italiano per lo Studio della Sopravvivenza nell'Infarto Miocardico). Ital Heart J 2000;1:810-20.

19. GISSI-HF investigators. Effect of rosuvastatin in patients with chronic heart failure (the GISSI-HF trial): a randomised, double-blind, placebo-controlled trial. Lancet 2008;372:1231-9.

20. Heart Protection Study Collaborative Group. MRC/BHF Heart Protection Study of cholesterol lowering with simvastatin in 20,536 high-risk individuals: a randomised placebo-controlled trial. Lancet 2002;360:7-22.

21. Ridker PM, Danielson E, Fonseca FA, et al. Rosuvastatin to prevent vascular events in men and women with elevated C-reactive protein. N Engl J Med 2008;359:2195-207.

22. LIPID Study Group (Long-term Intervention with Pravastatin in Ischaemic Disease). Long-term effectiveness and safety of pravastatin in 9014 patients with coronary heart disease and average cholesterol concentrations: the LIPID trial follow-up. Lancet 2002;359:1379-87.

23. Serruys PW, de Feyter $\mathrm{P}$, Macaya $\mathrm{C}$, et al, Lescol Intervention Prevention Study (LIPS) Investigators. Fluvastatin for prevention of cardiac events following successful first percutaneous coronary intervention: a randomized controlled trial. JAMA 2002;287:3215-22.

24. Nakamura $\mathrm{H}$, Arakawa $\mathrm{K}$, Itakura $\mathrm{H}$, et al. Primary prevention of cardiovascular disease with pravastatin in Japan (MEGA Study): a prospective randomised controlled trial. Lancet 2006;368:1155-63.
25. Shepherd J, Cobbe SM, Ford I, et al. Prevention of coronary heart disease with pravastatin in men with hypercholesterolemia. West of Scotland Coronary Prevention Study Group. N Engl J Med 1995;333:1301-7.

26. [No authors listed]. Randomised trial of cholesterol lowering in 4444 patients with coronary heart disease: the Scandinavian Simvastatin Survival Study (4S). Lancet 1994;344:1383-9.

27. Shepherd J, Blauw GJ, Murphy MB, et al. Pravastatin in elderly individuals at risk of vascular disease (PROSPER): a randomised controlled trial. Lancet 2002;360:1623-30.

28. Hayward RA, Kent DM, Vijan S, et al. Multivariable risk prediction can greatly enhance the statistical power of clinical trial subgroup analysis. BMC Med Res Methodol 2006;6:18.

29. Kent DM, Rothwell PM, Ioannidis JP, et al. Assessing and reporting heterogeneity in treatment effects in clinical trials: a proposal. Trials 2010;11:85.

30. Law MR, Wald NJ, Rudnicka AR. Quantifying effect of statins on low density lipoprotein cholesterol, ischaemic heart disease, and stroke: systematic review and meta-analysis. $B M J$ 2003;326:1423.

31. Bulbulia R, Bowman L, Wallendszus K, et al, Heart Protection Study Collaborative Group. Effects on 11-year mortality and morbidity of lowering LDL cholesterol with simvastatin for about 5 years in 20,536 high-risk individuals: a randomised controlled trial. Lancet 2011;378:2013-20.

32. Holman RR, Paul SK, Bethel MA, et al. 10-year follow-up of intensive glucose control in type 2 diabetes. $N$ Engl J Med 2008;359:1577-89.

33. Schooling CM, Freeman G, Cowling BJ. Mendelian randomization and estimation of treatment efficacy for chronic diseases. Am J Epidemiol 2013;177:1128-33.

34. Voight BF, Peloso GM, Orho-Melander M, et al. Plasma HDL cholesterol and risk of myocardial infarction: a Mendelian randomisation study. Lancet 2012;380:572-80.

35. Kathiresan S, Melander 0, Anevski D, et al. Polymorphisms associated with cholesterol and risk of cardiovascular events. $N$ Engl J Med 2008;358:1240-9.

36. Ference BA, Yoo W, Alesh I, et al. Effect of long-term exposure to lower low-density lipoprotein cholesterol beginning early in life on the risk of coronary heart disease: a Mendelian randomization analysis. J Am Coll Cardiol 2012;60:2631-9.

37. Cohen JC, Boerwinkle E, Mosley TH Jr, et al. Sequence variations in PCSK9, low LDL, and protection against coronary heart disease. $N$ Engl J Med 2006;354:1264-72.

38. Stitziel NO, Won HH, Morrison AC, Myocardial Infarction Genetics Consortium Investigators. Inactivating Mutations in NPC1L1 and Protection from Coronary Heart Disease. N Engl J Med 2014;371:2072-82.

39. Ference BA, Mahajan N. The role of early LDL lowering to prevent the onset of atherosclerotic disease. Curr Atheroscler Rep 2013;15:312.

40. Hayward RA, Heisler M, Adams J, et al. Overestimating outcome rates: statistical estimation when reliability is suboptimal. Health Serv Res 2007;42:1718-38.

41. Hutcheon JA, Chiolero A, Hanley JA. Random measurement error and regression dilution bias. BMJ 2010;340:c2289.

42. Navar-Boggan AM, Peterson ED, D'Agostino RB, et al. Hyperlipidemia in early adulthood increases long-term risk of coronary heart disease. Circulation 2015;131:451-8.

43. Kusters DM, Avis HJ, de Groot E, et al. Ten-year follow-up after initiation of statin therapy in children with familial hypercholesterolemia. JAMA 2014;312:1055.

44. Robinson JG, Gidding SS. Curing atherosclerosis should be the next major cardiovascular prevention goal. J Am Coll Cardiol 2014;63:2779-85.

45. Herman WH, Hoerger TJ, Brandle M, et al. The costeffectiveness of lifestyle modification or metformin in preventing type 2 diabetes in adults with impaired glucose tolerance. Ann Intern Med 2005;142:323-32. 
46. Vijan S, Sussman JB, Yudkin JS, et al. EFfect of patients' risks and preferences on health gains with plasma glucose level lowering in type 2 diabetes mellitus. JAMA Intern Med 2014;174:1227-34.

47. Zulman DM, Vijan S, Omenn GS, et al. The relative merits of population-based and targeted prevention strategies. Milbank $Q$ 2008;86:557-80.
48. Hayward RA, Krumholz HM, Zulman DM, et al. Optimizing statin treatment for primary prevention of coronary artery disease. Ann Intern Med 2010; 152:69-77.

49. Grade Definitions. U.S. Preventive Services Task Force, 2014. http://www.uspreventiveservicestaskforce.org/Page/Name/ grade-definitions (accessed 30 Mar 2015). 